\title{
AN ITERATION METHOD FOR THE DETERMINATION OF AN \\ UNKNOWN BOUNDARY CONDITION IN A PARABOLIC INITIAL-BOUNDARY VALUE PROBLEM
}

\author{
by MICHAEL PILANT* and WILLIAM RUNDELL*
}

(Received 20th May 1987)

\section{Introduction}

Consider the initial boundary value problem

$$
\begin{gathered}
u_{t}-u_{x x}=\gamma(x, t) \quad 0<x<1 \quad 0<t<T \\
u(x, 0)=u_{0} \quad 0<x<1 \\
u_{x}(0, t)=f(u(0, t)) \quad 0 \leqq t \leqq T \\
-u_{x}(1, t)=f(u(1, t)) \quad 0 \leqq t \leqq T
\end{gathered}
$$

In the context of the heat conduction problem, this models the case where the heat flux across the ends at the rod is a function of the temperature. If the heat exchange between the rod and its surroundings is purely by convection, then one commonly assumes that $f$ is a linear function of the difference in temperatures between the ends of the rod and that of the surroundings, (Newton's law of cooling). For the case of purely radiative transfer of energy a fourth power law for the function $f$ is usual, (Stefan's law).

We envision the situation where either the actual method of heat transfer is not known, or where it cannot be assumed that the governing laws have such a simple form, and would like to set up an experiment that would allow the recovery of the exact form of the function $f$. The major part of this paper will be devoted to showing that one can in fact recover the unknown $f$ by monitoring the temperature $u$ at one end of the rod for some time interval $0 \leqq t \leqq T$. That is we overpose the problem (1)-(4) by giving the additional boundary data

$$
u(0, t)=h(t)
$$

In a later part of the paper we shall discuss other possible forms for the boundary data in this model problem, and present some numerical examples to illustrate the feasibility of the method.

*Supported in part by AFOSR and NSF under grant number DMS-8701338. 


\section{Existence and uniqueness}

The central question to be answered in this paper is to find conditions under which one can find the solution pair $(u, f)$ to $(1)-(5)$, when $u_{0}(x)$ and $\gamma(x, t)$ are known.

We shall recall certain standard functions and state the properties that we require from them. The derivation of these properties can be found in [1].

Let $\theta(t)$ denote the theta function defined by

$$
\theta(x, t)=\sum_{m=-\infty}^{m=\infty} K(x-2 m, t) \quad t>0
$$

where $K(x, t)$ is the free space heat kernel

$$
K(x, t)=\frac{1}{\sqrt{4 \pi t}} e^{-x^{2} / 4 t} \quad-\infty<x<\infty, \quad t>0
$$

\section{Lemma 1.}

$$
\theta(-1, t)=\frac{1}{\sqrt{4 \pi t}} \sum_{k=1}^{\infty} e^{-(k-1 / 2)^{2} / t}
$$

is a $C^{\infty}$ function on $[0, \infty)$ whose nth partial derivative in $t$, evaluated at $t=0$, vanishes.

\section{Lemma 2.}

$$
\theta(0, t)=\frac{1}{\sqrt{4 \pi t}}+\frac{1}{\sqrt{\pi t}} \sum_{k=1}^{\infty} e^{-k^{2} / t}=\frac{1}{\sqrt{4 \pi t}}+H(t),
$$

where $H(t)$ is $C^{\infty}$ on $[0, \infty)$ with $H^{(n)}(0)=0$ for all $n$.

Let the functions $v(x, t)$ and $w(x, t)$ be the solutions of the initial boundary value problems

$$
\begin{gathered}
w_{\imath}-w_{x x}=\gamma(x, t) \quad 0<x<1, \quad 0<t<T \\
w(x, 0)=u_{0}(x) \quad 0 \leqq x \leqq 1 \\
w_{x}(0, t)=-w_{x}(1, t)=0 \quad 0 \leqq t \leqq T
\end{gathered}
$$

and

$$
\begin{gathered}
v_{t}-v_{x x}=\gamma(x, t) \quad 0<x<1 \quad 0<t<T \\
v(x, 0)=u_{0}(x) \quad 0 \leqq x \leqq 1
\end{gathered}
$$




$$
\begin{array}{cc}
v_{x}(0, t)=g_{0}(t) & 0 \leqq t \leqq T \\
-v_{x}(1, t)=g_{1}(t) & 0 \leqq t \leqq T
\end{array}
$$

for some functions $g_{0}(t)$ and $g_{1}(t)$.

In this case the function $v(x, t)$ has the representation [1],

$$
v(x, t)=w(x, t)-2 \int_{0}^{t} \theta(x, t-\tau) g_{0}(\tau) d \tau-2 \int_{0}^{t} \theta(x-1, t-\tau) g_{1}(\tau) d \tau
$$

and thus

$$
v(0, t)=w(0, t)-2 \int_{0}^{t} \theta(0, t-\tau) g_{0}(\tau) d \tau-2 \int_{0}^{t} \theta(-1, t-\tau) g_{1}(\tau) d \tau
$$

If $u_{0}(x), \gamma(x, t), g_{0}(t)$ and $g_{1}(t)$ are given, one can immediately determine $v(0, t)$. Can the function $g_{0}(t)$ be recovered from a knowledge of $v(0, t), u_{0}(x), \gamma(x, t)$ and $g_{1}(t)$ ? The answer is, of course yes and if we let

$$
k(t)=\frac{1}{2}[w(0, t)-v(0, t)]-\int_{0}^{t} \theta(-1, t-\tau) g_{1}(\tau) d \tau
$$

then $g_{0}(t)$ is the solution of the first kind Abel-type integral equation,

$$
\int_{0}^{1} \theta(0, t-\tau) g_{0}(\tau) d \tau=k(t)
$$

which can be written in the form

$$
\frac{1}{\sqrt{\pi}} \int_{0}^{t} \frac{g_{0}(\tau)}{\sqrt{t-\tau}} d \tau+\int_{0}^{t} H(t-\tau) g_{0}(\tau) d \tau=k(t)
$$

where $H(t)$ is $C^{\infty}$ for $t \geqq 0$, by Lemma 2 .

The proof of the next lemma can be found in $[1$, p. 85].

Lemma 3. If $k(t)$ is absolutely continuous, with $k^{\prime}(t) \in L^{\infty}$, then the integral equation (12) can be written in the form

$$
g_{0}(t)=\frac{2}{\sqrt{\pi}}\left\{\frac{k(0)}{\sqrt{t}}+\int_{0}^{t} \frac{k^{\prime}(\tau) d \tau}{\sqrt{t-\tau}}+\int_{0}^{t} S(t, \tau) g_{0}(\tau) d \tau\right\}
$$

where

$$
S(t, \tau)=\frac{H(0)}{\sqrt{t-\tau}}+\int_{\tau}^{t} \frac{1}{\sqrt{t-\eta}} H^{\prime}(\eta-\tau) d \eta
$$


Now let $v(0, t)=h(t)$ and set

$$
\begin{aligned}
& g_{0}(t)=f(u(0, t)) \\
& g_{1}(t)=f(u(1, t))
\end{aligned} \quad 0 \leqq t \leqq T
$$

so that

$$
k(t)=\frac{1}{2}[w(0, t)-h(t)]-\int_{0}^{t} \theta(-1, t-\tau) f(u(1, \tau)) d \tau
$$

Note that $H(0)=0$, and if $w(0,0)-h(0) \equiv u_{0}(0)-h(0)$ is zero (that is, the initial data and overposed data are compatible at $x=0, t=0$ ), then using (13) we obtain the integral equation

$$
\begin{aligned}
f(h(t))= & \frac{2}{\sqrt{\pi}} \int_{0}^{t} S(t, \tau) f(h(\tau)) d \tau \\
& +\frac{1}{\sqrt{\pi}} \int_{0}^{t}\left\{w_{\tau}(0, \tau)-h^{\prime}(\tau)-2 \int_{0}^{\tau} \theta_{\imath}(-1, \tau-\eta) f(u(1, \eta)) d \eta\right\} \frac{d \tau}{\sqrt{t-\tau}} .
\end{aligned}
$$

The right-hand side of (15) is a nonlinear function of $f$, since the final term contains $f(u(1, t))$ and $u(1, t)$ itself depends on the function $f$, that is $u \equiv u(x, t ; f)$.

Assuming that $h(t)$ is a one-to-one function, then by setting $f(t)=f(h(t))$, we obtain

$$
\begin{aligned}
f(t)=\frac{1}{\sqrt{\pi}} \int_{0}^{t} \frac{w_{\tau}(0, \tau)-h^{\prime}(\tau)}{\sqrt{t-\tau}} d \tau+\frac{2}{\sqrt{\pi}} \int_{0}^{t} S(t, \tau) f(\tau) d \tau \\
-\frac{2}{\sqrt{\pi}} \int_{0}^{t} \int_{0}^{\tau} \theta_{t}(-1, \tau-\eta) f\left(h^{-1}(u(1, \eta))\right) d \eta \frac{d \tau}{\sqrt{t-\tau}}
\end{aligned}
$$

and write this as

$$
f(t)=\mathbf{T}_{h}[f](t) .
$$

The space of functions of a single variable whose $m$ th derivative is Hölder continuous of exponent $\alpha$ on the interval $[a, b]$ we shall denote by $C^{m+\alpha}[a, b], 0<\alpha \leqq 1$ and endow this space with its usual norm. In particular, we shall be interested in the space of Lipschitz continuous functions on the interval $[0, T]$. We shall use the symbol $\mathscr{L}$ for this space and set

$$
|f|_{1}=\sup _{0 \leqq t_{1}<t_{2} \leqq T} \frac{\left|f\left(t_{1}\right)-f\left(t_{2}\right)\right|}{\left|t_{1}-t_{2}\right|}
$$

as the usual seminorm, and $\|\cdot\|_{1}=|\cdot|_{1}+\|\cdot\|_{\infty}$ as the norm. We will also need the space of 
functions with a half power fractional derivative on the interval $[0, T]$, and use the symbol $\mathscr{H}$ for this space, setting

$$
|f|_{\mathscr{P}}=\sup _{0 \leqq t_{1}<t_{2} \leqq T} \frac{\left|f\left(t_{1}\right)-f\left(t_{2}\right)\right|}{\left|t_{1}-t_{2}\right|^{1 / 2}}
$$

as the seminorm, and $\|\cdot\|_{\mathscr{X}}=|\cdot|_{\mathscr{X}}+\|\cdot\|_{\infty}$ as the norm.

We shall assume that the data $u_{0}(x), \gamma(x, t)$ and $h(t)$ satisfy:

A.1. $u_{0}(x) \in C^{2+1 / 2}[0,1]$

A.2. $\gamma(x, t)$ is Lipschitz continuous in $x$ and $C^{1 / 2}$ in $t$.

A.3. The overposed data is continuously differential of $[0, T]$ with $h^{\prime}(t) \in C^{1 / 2}[0, T]$; $h(t)$ is monotone on this interval and $h(0)=u_{0}(0)$.

Remark. The smoothness assumptions on these functions are sufficient to guarantee that $w_{t}(0, t)-h^{\prime}(t) \in C^{1 / 2}[0, T]$. The compatibility condition implies that $k(0)=0$ in (13).

We can rewrite the integral equation (16), in the form

$$
\tilde{f}=\mathbf{T}_{h}[\tilde{f}]=d(t)+\mathscr{I}_{1}[\tilde{f}]+\mathscr{A} \mathscr{I}_{2}\left[F_{h}(\tilde{f})\right]
$$

where $\mathscr{I}_{1}[f]$ and $\mathscr{I}_{2}[f]$ denote the linear operators of Volterra type

$$
\begin{gathered}
\mathscr{I}_{1}[f]=\frac{2}{\sqrt{\pi}} \int_{0}^{t} S(t, \tau) f(\tau) d \tau \\
\mathscr{I}_{2}[f]=-\frac{2}{\sqrt{\pi}} \int_{0}^{1} \theta_{t}(-1, t-\tau) f(\tau) d \tau
\end{gathered}
$$

and $\mathscr{A}[f]$ is the Abel operator

$$
\mathscr{A}[f]=\int_{0}^{t} \frac{f(\tau)}{\sqrt{t-\tau}} d \tau
$$

Here $F_{h}$ denotes the nonlinear mapping

$$
f \rightarrow f \circ h^{-1}(u(1, t ; f))
$$

and $u(1, t ; f)$ is the solution of $(1)-(4)$.

Lemma 4. If the function $\psi(\cdot)$ is Lipschitz continuous, that is $\left|\psi\left(x_{1}\right)-\psi\left(x_{2}\right)\right| \leqq$ $c\left|x_{1}-x_{2}\right|$, and $f_{1}$ and $f_{2}$ are in $\operatorname{Lip}[0, T]$, then for $i=1,2$

$$
\left\|\mathscr{S}_{i}\left[\psi\left(f_{1}\right)\right]-\mathscr{I}_{i}\left[\psi\left(f_{2}\right)\right]\right\|_{1} \leqq c \alpha_{i}(t)\left\|f_{1}-f_{2}\right\|_{\infty}
$$

where $\alpha_{i}(t)=O(t)$ as $t \rightarrow 0^{+}$. 
Proof. This follows directly by standard estimates and using the fact that each of the kernels in the operators $\mathscr{I}_{1}$ and $\mathscr{I}_{2}$ are continuous functions. Let us denote by $G$ the kernel of $\mathscr{I}_{i}$ for $i=1,2$.

$$
\begin{aligned}
\left|\mathscr{I}\left[f_{1}\right]-\mathscr{I}\left[f_{2}\right]\right|= & \left|\int_{0}^{t_{2}}\left\{G\left(t_{1}-\tau\right)-G\left(t_{2}-\tau\right)\right\}\left\{\psi\left(f_{1}\right)-\psi\left(f_{2}\right)\right\} d \tau\right| \\
& +\left|\int_{t_{2}}^{t_{1}} G\left(t_{1}-\tau\right)\left\{\psi\left(f_{1}\right)-\psi\left(f_{2}\right)\right\} d \tau\right| \\
\leqq & \left|\left(t_{2}-t_{1}\right)\right| \int_{0}^{t_{2}}\left|G_{t}(\xi-\tau)\right|\left|\psi\left(f_{1}\right)-\psi\left(f_{2}\right)\right| d \tau \\
& +\int_{t_{2}}^{t_{1}}\left|G\left(t_{1}-\tau\right)\right|\left|\psi\left(f_{1}\right)-\psi\left(f_{2}\right)\right| d \tau \\
\leqq & \alpha(t)\left|t_{2}-t_{1}\right||| \psi\left(f_{1}\right)-\psi\left(f_{2}\right) \|_{\infty}
\end{aligned}
$$

where $\alpha \rightarrow 0$ as $t \rightarrow 0$. Since each of the functions $S$ and $\theta_{t}(-1, t)$ and their derivatives vanish at $t=0$, which implies that $G$ vanishes with its derivative at $t=0$. The conclusion is then immediate.

Lemma 5. Under the same conditions of $f_{1}, f_{2}$ and $\psi$ we have

$$
\| \mathscr{A}\left[\psi\left(f_{1}\right)\right]-\mathscr{A}\left[\psi\left(f_{2}\right)\left\|_{\mathscr{\varkappa}} \leqq c\right\| f_{1}-f_{2} \|_{\infty}\right.
$$

In fact, it follows that the Abel integral operator maps functions in $C^{\alpha}(0, T)$ into functions in $C^{\alpha+1 / 2}[0, T]$.

We thus have

Lemma 6. If $u_{0}, \gamma$ and $h$ satisfy $A .1-A .3$, then the function

$$
d(t)=\mathscr{A}\left[w_{t}(0, t)-h^{\prime}(t)\right]
$$

lies in $\mathscr{H}[0, T]$.

Lemma 7. If $f_{1}$ and $f_{2}$ are Lipschitz functions of their independent variable, then the solution of

$$
\begin{gathered}
u_{t}^{(i)}-u_{x x}^{(i)}=\gamma(x, t) \quad 0<x<1, \quad 0<t<T \\
u^{(i)}(x, 0)=u_{0}(x) \quad 0 \leqq x \leqq 1 \\
u^{(i)}(0, t)=h(t) \quad 0 \leqq t \leqq T \\
-u_{x}^{(i)}(1, t)=f_{i}\left(u^{(i)}(1, t)\right)=f \circ h^{-1}\left(u^{(i)}(1, t)\right) \quad 0 \leqq t \leqq T
\end{gathered}
$$


for $i=1,2$, evaluated at $x=1$ satisfies

$$
\left\|u^{(1)}(1, t)-u^{(2)}(1, t)\right\|_{\varkappa} \leqq C\left\|f_{1}-f_{2}\right\|_{\infty}
$$

for $T$ sufficiently small, and some constant $C$ depending on $T$ and the Lipschitz norms of $f_{1}$ and $f_{2}$.

Proof. The difference $u^{(1)}-u^{(2)}$ can be written in the form

$$
u^{(1)}(1, t)-u^{(2)}(1, t)=\int_{0}^{t} L(t-\tau)\left[f_{1} \circ h^{-1}\left(u^{(1)}(1, \tau)\right)-\tilde{f}_{2} \circ h^{-1}\left(u^{(2)}(1, \tau)\right)\right] d \tau
$$

for some kernel $L(\tau)$, with a square root singularity at the origin.

Let $\phi(t)=u^{(1)}(1, t)-u^{(2)}(1, t)$. Then by breaking up the interval of integration we obtain

$$
\begin{aligned}
\phi\left(t_{1}\right)-\phi\left(t_{2}\right)= & \int_{0}^{t_{2}}\left\{L\left(t_{1}-\tau\right)-L\left(t_{2}-\tau\right)\right\}\left\{f_{1}\left(u^{(1)}(1, \tau)\right)-f_{1}\left(u^{(2)}(1, \tau)\right)\right\} d \tau \\
& +\int_{0}^{t_{2}}\left\{L\left(t_{1}-\tau\right)-L\left(t_{2}-\tau\right)\right\}\left\{f_{1}\left(u^{(2)}(1, \tau)\right)-f_{2}\left(u^{(2)}(1, \tau)\right)\right\} d \tau \\
& +\int_{t_{2}}^{t_{1}} L\left(t_{1}-\tau\right)\left\{f_{1}\left(u^{(1)}(1, \tau)\right)-f_{1}\left(u^{(2)}(1, \tau)\right)\right\} d \tau \\
& +\int_{t_{2}}^{t_{1}} L\left(t_{1}-\tau\right)\left\{f_{1}\left(u^{(2)}(1, \tau)\right)-f_{2}\left(u^{(2)}(1, \tau)\right)\right\} d \tau \\
\equiv & I_{1}+I_{2}+I_{3}+I_{4} .
\end{aligned}
$$

Now,

$$
\begin{aligned}
\left|I_{1}\right| & \leqq \int_{0}^{t_{2}}\left|L\left(t_{1}-\tau\right)-L\left(t_{2}-\tau\right)\left\|f_{1}\right\| u^{(1)}(1, \tau)-u^{(2)}(1, \tau)\right| d \tau \\
& \leqq\left.\left|f_{1}\right|\right|_{1}\left\|u^{(1)}-u^{(2)}\right\|_{\infty} \int_{0}^{t_{2}} \int_{t_{2}}^{t_{1}}\left|L_{r}(s-\tau)\right| d s d \tau \\
& \leqq C_{1} \sqrt{t_{2}-t_{1}}\left|f_{1}\right|_{1}\left\|u^{(1)}-u^{(2)}\right\|_{\infty} .
\end{aligned}
$$

For $I_{2}$ we have

$$
\begin{aligned}
\left|I_{2}\right| & \leqq \int_{0}^{t_{2}}\left|L\left(t_{1}-\tau\right)-L\left(t_{2}-\tau\right)\right|\left|f_{1}-f_{2}\right| d \tau \\
& \leqq C_{2} \sqrt{t_{2}-t_{1}}\left\|f_{1}-f_{2}\right\|_{\infty} .
\end{aligned}
$$


For $I_{3}$ and $I_{4}$ we have

$$
\begin{aligned}
\left|I_{3}\right| & \leqq\left.\int_{i_{1}}^{t_{2}}|L(\tau)|\left|f_{1}\right|\right|_{1}|| u^{(1)}(1, \cdot)-u^{(2)}(1, \cdot) \|_{\infty} d \tau \\
& \leqq C_{3} \sqrt{t_{2}-t_{1}}\left|f_{1}\right|_{1} \mid\left\|u^{(1)}-u^{(2)}\right\|_{\infty} \\
\left|I_{4}\right| & \leqq \int_{t_{1}}^{t_{2}}|L(\tau)|\left|f_{1}-f_{2}\right|_{1} d \tau \\
& \leqq C_{4} \sqrt{t_{2}-t_{1}}\left\|f_{1}-f_{2}\right\|_{\infty} .
\end{aligned}
$$

Combining these above inequalities we obtain

$$
|\phi|_{\mathscr{H}} \leqq C_{5}\left(\|\phi\|_{\infty}+\left\|f_{1}-f_{2}\right\|_{\infty}\right)
$$

where $C_{5}$ is independent of $T$. Noting that $\phi(0)=0$, we have $\|\phi\|_{\infty} \leqq \sqrt{t}|\phi|_{\mathscr{H}}$. This yields

$$
|\phi|_{\mathscr{H}} \leqq C_{5}\left(\sqrt{t}\|\phi\|_{\mathscr{H}}+\left\|f_{1}-f_{2}\right\|_{\infty}\right)
$$

and consequently,

$$
\|\phi\|_{\mathscr{H}} \leqq \frac{C_{5}}{1-C_{5} \sqrt{T}}\left\|f_{1}-f_{2}\right\|_{\infty}
$$

and the conclusion of the lemma follows.

Compatibility of the data at $t=0$ ensures that $f_{1}(h(0))=f_{2}(h(0))$, and therefore $\left\|\tilde{f}_{1}-\tilde{f}_{2}\right\|_{\infty} \leqq \sqrt{t}\left|\tilde{f}_{1}-\tilde{f}_{2}\right|_{\varkappa}$. Lemmas $4-7$ now give that

$$
\left\|\mathbf{T}_{h}\left[\tilde{f}_{1}\right]-\mathbf{T}_{h}\left[\tilde{f}_{2}\right]\right\|_{\mathscr{H}} \leqq \frac{C_{5} \sqrt{T}}{1-C_{5} \sqrt{T}}\left\|\tilde{f}_{1}-\tilde{f}_{2}\right\|_{\mathscr{H}}
$$

and thus for $T$ sufficiently small $(2 C \sqrt{T}<1), \mathbf{T}_{h}[]$ is a contraction on $\mathscr{H}$.

In order to show that $T_{h}[]$ has a fixed point we require that the function $h^{-1}(u(1, t, f))$ be in the domain of $f$ (that is $u(1, t, f)$ be in the domain of $f$ ). In other words, we may only recover $f(u)$ over a range $U_{0} \leqq u \leqq U_{T}$ if the overposed data $h(t)$ contains this set of values for $0 \leqq t \leqq T$.

We thus assume that

A.4. For each $t, 0 \leqq t \leqq T$, the function $u(1, t, f)$ lies in the interval $[h(0), h(t)]$.

Physically this condition says that the range of values of temperature on the hidden boundary $x \doteq 1$ is contained in the range of values at temperature on the boundary where this quantity can be measured. 
One cannot guarantee A.4 solely by giving conditions on the data $u_{0}(x), \gamma(x, t)$ and $h(t)$, for it will depend on the unknown function $f$. There are, however, easily obtainable conditions under which A.4 can be made to hold. For example, if it is known a priori that $f \geqq 0$, (cooling on the boundary), that $\gamma \leqq 0$ (no internal sources), and $u_{0}(0)=u_{0}(1)$ then it is easy to give conditions under which $h(t)$ will be a decreasing function and $h(t)=u(0, t) \leqq u(1, t) \leqq u_{0}(0)=u_{1}(0)=h(0)$ for all $t \geqq 0$. If $f>0$ for $u>0$ with $f(0)=0$ then $u(x, t) \rightarrow 0$ as $t \rightarrow \infty$ for all $x$ and thus both $u(0, t)=h(t)$ and $u(1, t)$ will be contained in the interval $\left(0, u_{0}(0)\right)$ for all $t>0$.

One of the advantages of using the contraction mapping is to allow us to give a constructive algorithm for the recovery of $f$. In order to apply the associated iteration procedures we must ensure that each successive iterate lies in the correct range and we do this by extending the domain of $f=f(h(t))$ to the whole line by extending $f$ to be constant outside of $[0, T]$. The extended function will remain Lipschitz.

We have proved the following theorem.

Theorem 1. Let A.1-A.4 hold. Then there exist a unique solution pair $(u, f)$ to $(1.1)$ to (1.5) over the interval $0 \leqq t \leqq t^{*}$, for some $t^{*}>0$.

Several factors may allow us to extend this above conclusion of Theorem 1 to global values of time. The constant $C$ appearing in (20) will in general depend on (the Lipschitz) norms at the function $u$. If the allowable class of functions $f$ is restricted to the uniformly Lipschitz functions, this will be the case. If $f$ is restricted to be nonnegative with $f(0)=0$, then there will be a heat loss from the rod for all $t>0$, and the maximum principle shown that $u(x, t)$ remains uniformly bounded by the maximum at the initial time, provided there are no sources, that is $\gamma(x, t) \leqq 0$. These two cases encompass many of the problems of physical interest.

\section{Related problems}

Some obvious extensions of the method can be made. For example the boundary conditions can be taken in the form

$$
u_{x}(0, t)=\alpha_{0}(t) f(u(0, t))+\beta_{0}(t) \quad-u_{x}(1, t)=\alpha_{1}(t) f(u(1, t))+\beta_{1}(t)
$$

where $\alpha_{0}, \alpha_{1}, \beta_{0}$ and $\beta_{1}$ are known functions.

We can also consider the case when the radiation condition $u_{x}=f(u)$ hold at only one of the boundaries, while at the other, either the function $u$, or its normal derivative, is prescribed. In the latter case we look for the solution pair $(u, f)$ to the initial-boundary value problem

$$
\begin{gathered}
u_{t}-u_{x x}=\gamma(x, t) \quad 0<x<1, \quad 0<t<T \\
u(x, 0)=u_{0}(x) \quad 0 \leqq x \leqq 1 \\
u_{x}(0, t)=f(u(0, t)) \quad 0 \leqq t \leqq T
\end{gathered}
$$




$$
u_{x}(1, t)=g_{1}(t) \quad 0 \leqq t \leqq T
$$

If we give the overposed data at the boundary $x=0$

$$
u(0, t)=h(t) \quad 0 \leqq t \leqq T
$$

then the solution to (23)-(26) with (27), can be determined immediately. To see this, note that there is a unique solution $u(x, t)$ to (23), (24), (26) and (27). By evaluating the $x$-derivative of this function on the boundary $x=0$ we obtain the equation

$$
u_{x}(0, t)=f(h(t)) \quad 0<t<T
$$

from which $f(\cdot)$ can be determined if $h(t)$ is a monotone function of $t$.

For the problem (1)-(4), if $u(x, t)$ is symmetric about the midpoint of the interval, that is $u(x, t)=u(1-x, t)$ then by noting that $u_{x}\left(\frac{1}{2}, t\right)=0$, we can convert this case to the above problem. Such a symmetric condition would, for example, arise from a homogeneous equation with constant initial temperature.

If both the temperature and the flux is prescribed at the boundary $x=0$, that is,

$$
\begin{gathered}
u_{t}-u_{x x}=\gamma(x, t) \quad 0<x<1, \quad 0<t<T \\
u(x, 0)=u_{0}(x) \quad 0 \leqq x \leqq 1 \\
-u_{x}(1, t)=f(u(1, t)) \quad 0 \leqq t \leqq T \\
u_{x}(0, t)=g(t) \quad 0 \leqq t \leqq T \\
u(0, t)=h(t) \quad 0 \leqq t \leqq T
\end{gathered}
$$

then the solution to (29)-(32) can be written in the form

$$
u(x, t)=\widetilde{\psi}(x, t)+\int_{0}^{t} K_{1}(x, t-\tau) f(\theta(\tau)) d \tau
$$

where $\psi(x, t)$ is a known function depending on $u_{0}(x), g(t)$ and $\gamma(x, t)$. By evaluating (34) on the boundary $x=0$, and using (33), we obtain an integral equation for the unknown function $f(\cdot)$. Unfortunately this is a first kind Volterra equation with a smooth kernel, $\left(K_{1} \equiv \theta(0, t)\right.$ which is a $C^{\infty}$ function with $K_{1}^{(n)}(0)=0$ for all $\left.n\right)$. The conditions (32), (33) represent characteristic Cauchy data for the parabolic equation (29). Given sufficient restrictions on the functions $g$ and $h$, we can continue the function $u(x, t)$ as a solution of (29) into the region $x>0$, (we must somehow ensure compatibility with the initial condition (30)). By evaluating this function and its $x$ derivative on the line $x=1$, we can in theory recover the function $f$. This of course will lead to a severely ill-conditioned problem, as was suggested by the form of the integral equation (34). We thus see that the problem (29)-(34) will admit at most one solution pair $(u, f)$, but existence can only 
be guaranteed by making extremely restrictive (and difficult to determine) conditions on the data. Even if this can be done, continuous dependence of the solution on the data cannot be expected in any reasonable norm.

\section{Numerical examples}

We shall describe a numerical implementation of the solution of the integral equation (16) by using an iteration scheme.

The recipe for the procedure is as follows:

(a) Generate "experimental data". Select a function $f_{\text {act }}$ and obtain the solution $u\left(x, t ; f_{\text {act }}\right)$ to $(1)-(4)$. The value of this function on the line $x=0$ forms the overposed data, and is passed to the inversion routine as $h(t)$ at discrete points $t_{j}$.

(b) We are free to use the data in any order, and the derivation that led to (16) required us to solve equation (1) with the initial value (2), the condition at $x=1,(4)$, and the value of $u$ at $x=0,(5)$. We then use equation (3) as an "update scheme" for the iteration. That is, for a given function $f$, we solve (1), (2), (4) and (5) to find $u(x, t ; f)$. The mapping $\mathbf{T}_{h}[f]$ is now equivalent to

$$
\mathbf{T}_{h}[f]=u_{x}(0, t ; f)
$$

and this function can easily be found by direct differentiation of $u(x, t ; f)$.

(c) We thus generate an iteration scheme by choosing an initial function $f_{0}$, and obtaining successive iterates by

$$
f_{n+1}(t)=f_{n+1}(h(t))=\mathrm{T}_{h}\left[f_{n}\right] \equiv u_{x}^{(n)}\left(0, t ; f_{n}\right)
$$

where $u^{(n)}\left(x, t ; f_{n}\right)$ denotes the solution of (1), (2), (4) and (5) with $f=f_{n}$.

(d) The iteration is terminated when $\left\|f_{n}-f_{n+1}\right\|$ is within the desired accuracy.

This scheme is similar to that applied by the authors to recover nonlinear coefficients and source terms in elliptic and parabolic equations [2-5]. The central idea in these papers was to evaluate the differential operator on that section of the boundary where the overposed data is prescribed, and to use this to form an update algorithm for succesive approximations. For the problem (1)-(5), this is provided by the evaluation on $x=0$ of the boundary operator $u_{x}=f(u)$.

There are some remarks to be made on the above procedure.

(1) The forwards solver method used to generate $u(x, t ; f)$ from a given function $f$, was a 3 time level finite difference scheme that was second order accurate in both $x$ and $t$.

(2) To obtain the data in part (a) above we used a course grid, $\Delta x=0.1, \Delta t=0.05$ being typical. This is to simulate the inevitable inaccuracies in measurement of real data. For the calculation of the solution $u(x, t ; f)$ of the direct problem in the inverse iteration scheme we used a finer mesh, with variable grid size to more accurately calculate the value of $u_{x}$ on the boundary $x=0$.

(3) The number of iterations required to obtain a given accuracy of $f$ will obviously 
depend on the tolerance required, but in all cases effective convergence was obtained within a few iterations. We illustrate the procedure with two examples, one for a cooling problem and the other for the case when the body is absorbing heat.

Example 1. Let $u(x, t)$ satisfy,

$$
\begin{gathered}
u_{t}-u_{x x}=3-2 x \quad 0<x<1, \quad 0<t<1 \\
u(x, 0)=0 \quad 0 \leqq x \leqq 1 \\
u_{x}(0, t)=f(u(0, t)) \quad 0 \leqq t \leqq T \\
-u_{x}(1, t)=f(u(1, t)) \quad 0 \leqq t \leqq T
\end{gathered}
$$

with $f_{a c r}(u)=e^{-u}-1$. The table below shows the difference of $f$ and $f_{a c t}$ in the supremum and $L^{2}$ norms until the difference in $\left\|f_{n+1}-f_{n}\right\|$ no longer decreased. The initial guess was $f_{0}=0$.

\begin{tabular}{ccc}
\hline \multicolumn{3}{c}{ Convergence rate of $f_{n}$ to $f_{\text {act }}$} \\
\hline$n$ & $\left\|f_{\text {act }}-f_{n}\right\|_{\infty}$ & $\left\|f_{\text {act }}-f_{n}\right\|_{L^{2}}$ \\
\hline 0 & 1.00000 & 1.47584 \\
1 & 0.72147 & 0.50049 \\
2 & 0.36802 & 0.18695 \\
3 & 0.08205 & 0.03202 \\
4 & 0.00855 & 0.00258 \\
5 & 0.00158 & 0.00078 \\
6 & 0.00157 & 0.00075 \\
\hline
\end{tabular}

Example 2. Let $u(x, t)$ satisfy,

$$
\begin{array}{cc}
u_{t}-u_{x x}=0 \quad 0<x<1, & 0<t<1 \\
u(x, 0)=1+2 x-3 x^{2}+x^{3} & 0 \leqq x \leqq 1 \\
u_{x}(0, t)=2 f(u(0, t)) & 0 \leqq t \leqq T \\
-u_{x}(1, t)=f(u(1, t)) & 0 \leqq t \leqq T
\end{array}
$$

with $f_{\text {act }}(u)=-u^{2}$.

The choice of initial data was simply to be compatible with the boundary data at $x=0$ and $x=1$. Note that the function $f$ is not sublinear, but its Lipschitz constant remains uniformly bounded since by the maximum principle the function $u(x, t)$ satisfies $0<u(x, t ; f) \leqq \sup u_{0}(x)$. 
The table below shows the difference of $f$ and $f_{a c t}$ in the supremum and $L^{2}$ norms until the difference in $\left\|f_{n+1}-f_{n}\right\|$ no longer decreased. The initial guess was $f_{0}=0$.

\begin{tabular}{ccc}
\hline \multicolumn{3}{c}{ Convergence rate of $f_{n}$ to $f_{\text {act }}$} \\
\hline$n$ & $\left\|f_{\text {act }}-f_{n}\right\|_{\infty}$ & $\left\|f_{\text {act }}-f_{n}\right\|_{L 2}$ \\
\hline 0 & 1.00000 & 0.44677 \\
1 & 0.18082 & 0.21749 \\
2 & 0.04051 & 0.02795 \\
3 & 0.00559 & 0.00476 \\
4 & 0.00559 & 0.00442 \\
5 & 0.00558 & 0.00442 \\
\hline
\end{tabular}

\section{REFERENCES}

1. J. R. Cannon, The one-dimensional heat equation, Encyclopedia Math. Appl. 23.

2. M. Pilant and W. Rundell, An inverse problem for a nonlinear parabolic equation, Comm. Partial Differential Equations 11 (4) (1986), 445-457.

3. M. Pilant and W. Rundell, Undetermined coefficient problems for nonlinear elliptic and parabolic equations, Internat. Ser. Numer. Math. 77 (1986), 139-154.

4. M. Pilant and W. Rundell, An inverse problem for a nonlinear elliptic equation, SIAM $J$. Math. Anal., 18 (6) (1987), 1801-1809.

5. M. Pilant and W. Rundell, Iteration schemes for unknown coefficient problems arising in parabolic equations, Numer. Methods in PDE, 3 (1987), 313-325.

Department of Mathematics

TEXAS A \& M UnIVERSITY

College Station TX 77843

USA 